Penrose's work, therefore, opens a new and fruitful approach to the study of paramagnetism.

The hyperfine structure of paramagnetic resonances may readily be understood if one imagines that in a stationary state the ion exerts a magnetic field $(H e)$ on the nucleus. The nucleus takes up quantized orientations of different magnetic energies in this field. The transitions observed in the paramagnetic resonance of cupric salts, in which the ionic spin has the value $\frac{1}{2}$, correspond to a reversal of spin and consequently of $\mathrm{He}$, the nuclear orientation remaining unchanged. The nuclear magnetic energy, therefore, changes sign, and there is a corresponding change in the quantum energy of the transition. The hyperfine splitting is clearly proportional to $H e$, provided the external field is strong enough to decouple the ionic moment from the field of the nucleus.

The field $H e$ arises partly from the spin magnetic moment of the electrons and partly from their orbital motion. In Polder's theory, the ion is supposed to be in an electric field of tetragonal symmetry which partly quenches the orbital moment. On this basis we calculate that:

(1) When the external magnetic field is parallel to the tetragonal axis

$$
H e=\frac{\beta}{r^{3}}\left[-\frac{4}{7}+g_{11}-2\right] \text { approximately. }
$$

(2) When the external field is perpendicular to the tetragonal axis :

$$
H e=\frac{\beta}{r^{3}}\left[\frac{2}{7}+g_{1}-2\right] \text { approximately. }
$$

In this expression small corrections which will not affect the following conclusions have been omitted. $\beta$ is the Bohr magneton, and $r$ is a suitable average radius of the $3 d$ magnetic state of the cupric ion.

The observed $g$-values are roughly $g_{11}=2 \cdot 40$ and $g_{\perp}=2 \cdot 10$, though there are definite deviations from tetragonal symmetry and the values differ slightly from salt to salt. This gives

$$
\frac{(H e)_{11}}{(H e)_{\perp}}=-\frac{0.17}{0.39}
$$

from which it follows that the hyperfine splitting should be least along the tetragonal axis. Penrose's measurements were made with the field varying between the perpendicular direction and about halfway towards the parallel direction, and show a variation in the opposite sense.

$$
\begin{aligned}
& \text { A. Abragam } \\
& \text { M. H. L. PRYce }
\end{aligned}
$$

Clarendon Laboratory, Oxford.

Mav 18.

\section{Critical-Frequency Difference Variations and the Poynting Vector in the lonosphere}

VíEASUREMENTS of the $F_{2}$-layer critical-frequency difference, $f x-f^{0}$, have now been made for the hourly records taken at Churchill, Manitoba, in 1945 and 1946 and at Portage la Prairie, Manitoba, in 1947 and 1948. These measurements corroborate results ${ }^{1}$ already reported for Clyde River, Baffin Land, in that large diurnal variations in the apparent magnetic field are found. However, the calculation assumes that ordinary and extraordinary modes are reflected from the same region of the ionosphere.
The direction of energy flow has been calculated for the ordinary and extraordinary modes by considering the average Poynting vector. The total deflexion of the ordinary mode northward and of the extraordinary mode southward (in the northern hernisphere) has been found for different wave frequencies, latitudes and collision frequencies, assuming a parabolic distribution of ionization and vertical incidence.

It is found that both ordinary and extraordinary modes suffer a small westward deflexion when collision is taken into account.

The horizontal north-south distance separating the points of reflexion of the ordinary and extraordinary modes increases as the critical frequency is reduced, assuming a constant half-thickness of the parabolic region. But if a diurnal expansion and contraction of the $F$-region exists, a corresponding increase and decrease of the north and south deflexions will occur. The resulting diurnal variation in the horizontal distance between the points of reflexion of the ordinary and extraordinary modes, together with the normal gradient of ionization with latitude, furnishes a mechanism which can explain the measured variations in the $f^{x}-f^{0}$ critical frequency difference.

Radio Propagation Laboratory, JAMES C. W. SCOTT

Defence Research Board,

Ottawa.

Jan. 8.

${ }^{1}$ Scott, J. C. W., Terr. Mag., 53 (June 1948).

\section{Slope of the Ocean}

For the component of steady flow at right angles to the pressure gradient on a rotating globe, the hydrodynamical equation may be written.

$$
u=-\frac{1}{2 \rho \omega \sin \varphi} \cdot \frac{\partial p}{\partial y}+F,
$$

where $u$ is the velocity of flow, $\partial p / \partial y$ the pressure gradient, $\varphi$ latitude, $\rho$ density, $\omega$ the angular velocity of rotation of the earth, and $F$ any extraneous forces apart from pressure, for example, that due to wind friction at the surface of the ocean. In a paper on the effect of wind on ocean currents ${ }^{1}$, I found empirically that the surface current due to wind flowed on the average at an angle of about $45^{\circ}$ to the wind with a velocity $\frac{7.9 \times 10^{-3}}{\sqrt{\frac{\sin \varphi}{4}}}$ that of the wind generating the current.

It is possible to divide the surface current, $u$, in equation (1) into two parts, $u_{1}$ due to the pressure gradient $\partial p / \partial y$, and $u_{2}$ due to the wind friction $F$. Then, from the relation above, allowance can be made for the wind friction, and so we get

$$
u_{1}=-\frac{1}{2 \rho \omega \sin \varphi} \frac{\partial p}{\partial y} \text {. }
$$

This gives directly the pressure gradients in the ocean surface, from which can be deduced the slope of the ocean (related to a level surface) if we know the mean flow of the current and of the wind.

In the Marine Branch of the Meteorological Office, there are calculations of the vector mean wind flow and the vector mean surface currents for the oceans of the world for a long period of years which are eminently suitable for making such a ealculation. 